\title{
Simulation Investigation over 1680km WDM Transmission System Reviewing NRZ and RZ Modulation Formats
}

\author{
Abdul Gafur ${ }^{1}$ \\ ${ }^{1}$ International Islamic University Chittagong, Dept. of Electronics and Telecommunications Engineering
}

\begin{abstract}
The investigation of a 16x10Gbps WDM transmission system on $0.8 \mathrm{~nm}$ channel spacing over $1680 \mathrm{~km}$ optical link distance with Single Mode Fiber (SMF) is predicted. Two different modulation techniques namely NRZ and RZ are examined. It is analyzed the BER, Q values and eye opening factor for this model using two different modulation formats. The simulation results show the dominance of WDM transmission exploiting NRZ encoding technique for long-haul optical transmission system. It is found that $\mathrm{Q}$ penalty is $0.68 \mathrm{~dB}$ for channel 1 and $0.24 \mathrm{~dB}$ for channel 8 considering eye diagrams with respect to NRZ and RZ modulation formats.
\end{abstract}

Keywords: - WDM, EDFA, SMF, DCF, BER, Q Factor, Eye opening factor

\section{INTRODUCTION}

Considering data rates, WDM transmission system is going to be prosperous and popular system which delivers abundant bandwidth with a comb of WDM channels [1-2].Consequently, still selection of an appropriate modulation technique is an open significant issue to maintain the high-quality of long haul WDM transmission performance [3-4].Modulation scheme also determines the properties of the optical signal during the propagation of light through the optical link [5]. As a result, it improves the bit rate as well as offers higher spectral efficiency.

In the last few years, different modulation techniques have been experimented to propagate the optical signal through optical link [6-8].At present, maximum optical distance with proper selection of modulation schemes is the key research interest factor to optimize the existing optical network and provide the high performance of the WDM transmission networks [9].In the optical link, EDFA has a large gain bandwidth, which is adequate to amplify the data channels with highest data rates [10].Thus, EDFA gain is flattened and plays an important role for long haul multi-channel light wave transmission system especially for WDM system [11].

Using low data rate, it is noticeable to reach the significant transmission distance in WDM system [12]. Moreover utilizing alternating fiber segments of opposite dispersion values which is a key method that keeps the total accumulated the dispersion values low while it suppresses the most non- liner effects [13].

In the receiver section of WDM system, the threshold detection technique is used where errors arise when noise in the system pushes a zero above and threshold pulls a one signal level below threshold at the sampling point. Due to the random nature of this noise, the total signal can be below threshold for a " 1 " and above threshold for a " 0 " which results in a non-zero probability of errors occurring with an associated Bit Error Rate (BER) [14-15].

Hence, the NRZ modulation process is possible if the bit stream is sent as simply the presence or absence of light on the fiber. This format of modulation is used for slow speed of optical links but has been replaced by other modulation techniques for most purposes [16].

Besides, considering the RZ modulation technique the signal returns to the zero state for every bit time period. There are two different line states required to represent a bit in a restricted bandwidth and this type of coding is not desired [16].

The aim of this paper is to analyze the effects of long haul WDM transmission system using NRZ and RZ modulation schemes to detect the effects of $1680 \mathrm{~km}$ optical link distance considering 20 numbers of spans using SMF [17].Simulation is performed using OptiSystem simulator to measure the variation between NRZ and RZ modulation formats in receiver section of the system. NRZ modulation scheme is introduced to get better performance of the long haul WDM transmission. The penalty between NRZ and RZ modulation schemes is simulated considering total optical link distance, Q factor and BER.

The paper is presented as follows. Model structure of long haul WDM transmission is presented in section II. Simulation results are described in Section III. Comments are placed in Section IV.

\section{MODEL STRUCTURE}

Simulation arrangements are constructed with Optisystem simulator version 7 [18].In Fig.1, the experimental setup is used for simulation. In this experiment two types of modulation methods are used which 
are NRZ and RZ respectively. NRZ has a good resistance of residual chromatic dispersion for EDFA and DCF of the optical link and shows less resistance for group velocity dispersion (GVD-SPM) compared to RZ complements [19].Mach-Zehnder modulator is connected with NRZ and RZ pulse generator in Fig.2 and Fig.3 respectively. In both encoding setups, the laser source is connected with the Mach-Zehnder modulator. A Pseudo Random Bit Sequence (PRBS) generator is encoded with both NRZ and RZ separately.

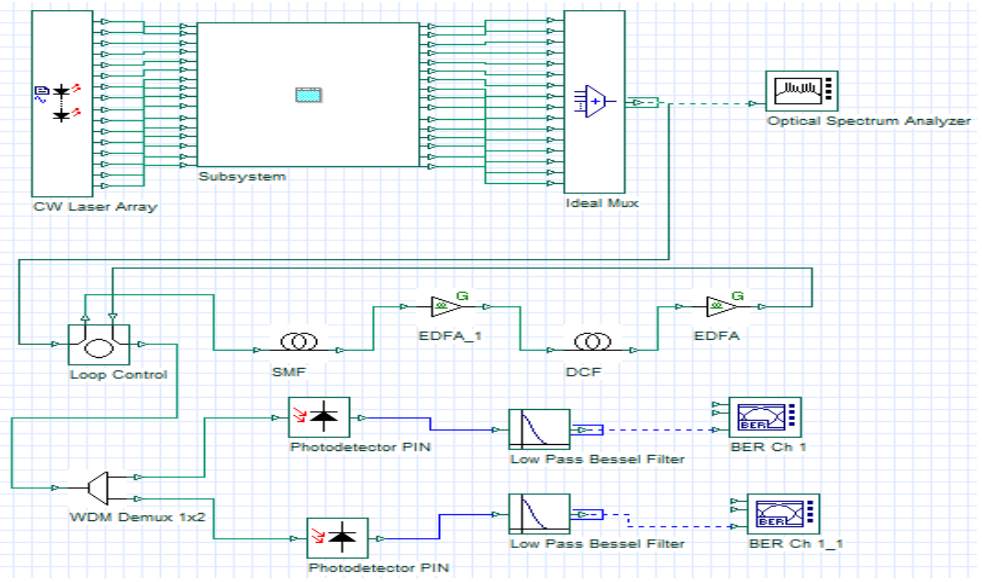

Fig.1: Long haul WDM communication system

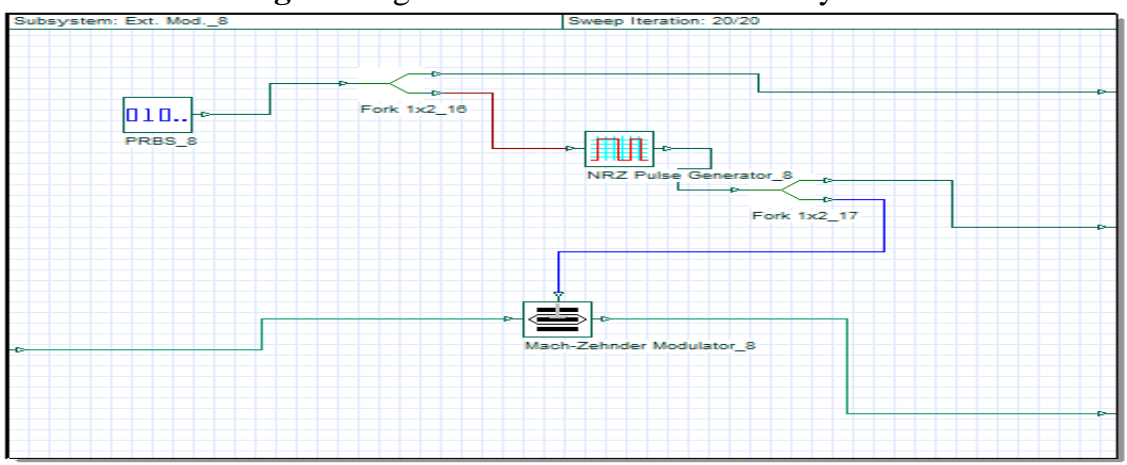

Fig.2: Signal Encoding using NRZ modulation technique

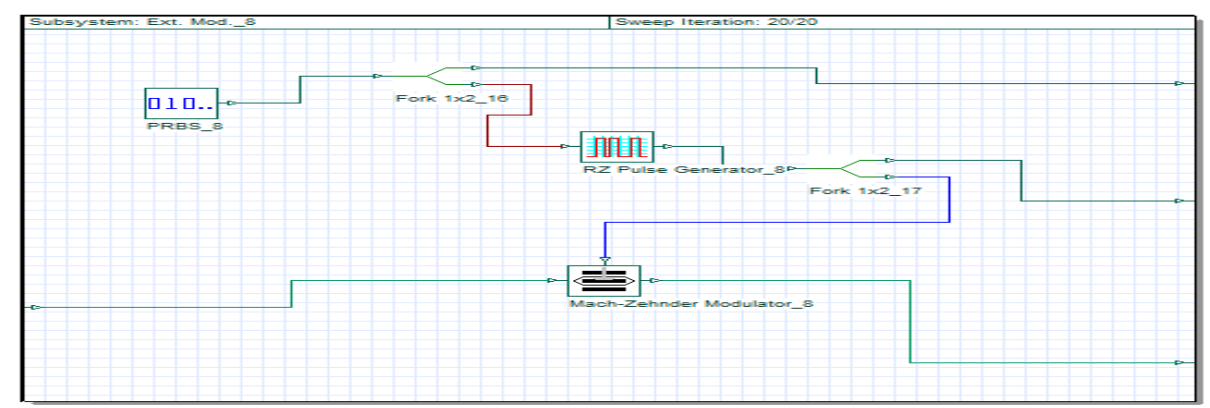

Fig.3: Signal Encoding using RZ modulation technique

The input signal is modulated using Mach-Zehnder modulator with Continuous Wave Laser (CWLaser) of different wavelengths. The signals are sent through the WDM multiplexer and transmitted over the optical fiber. SMF of $70 \mathrm{~km}$ long span is used in the optical link of the system. As a result, to compensate the amount of dispersion, the signal dispersion compensated fiber (DCF) of $14 \mathrm{~km}$ long is used. Each fiber span is followed by an optical amplifier with a noise figure of $6 \mathrm{~dB}$ to compensate the fiber loss. Loop control equipment is used in the system which has 20 loops which repeats the fiber spans of 20 times and calculate total optical link distance of $1680 \mathrm{~km}$ including the DCF.

The results are measured for the first channel which is the most affected channel of the system. Then the de-multiplexed signal goes through a PIN photo detector. After that a low pass filter is used to attenuate the unwanted signals. The output of the low pass filter is fed to a BER analyzer. 
The SMF has a dispersion of $17 \mathrm{ps} / \mathrm{nm}-\mathrm{km}$ and the dispersion compensation fiber has a negative dispersion of $-85 \mathrm{ps} / \mathrm{nm}-\mathrm{km}$. That's why the ratio between SMF and DCF is 5:1. The SMF has a loss of $0.2 \mathrm{~dB} / \mathrm{km}$. Consequently the gain of the EDFA is set at $14 \mathrm{~dB}$. The DCF has a loss of $0.5 \mathrm{~dB} / \mathrm{km}$. The second EDFA gain is set at 7dB. Furthermore, the simulation shows a distance of $1680 \mathrm{~km}$ with the inclusion of dispersion compensation fiber.

In this simulation, calculation of the total optical link distance considering the dispersion compensated fiber type is presented. In the receiver section of the model structure, a 1x2 WDM de-multiplexer is used which is connected to PIN photodiode to convert the optical signal to electrical signal. Low-pass Bessel filter is also connected with PIN photodiode to reconstruct the original signal [20].

Table 1: Values used in Long haul WDM communication system

\begin{tabular}{|c|c|}
\hline Parameters & Values \\
\hline Wavelength & $1554.4 \mathrm{~nm}-1566.5 \mathrm{~nm}$ \\
\hline Signal Input Power & $-10 \mathrm{dBm}$ \\
\hline length of SMF & $70 \mathrm{~km}$ \\
\hline length of DCF & $14 \mathrm{~km}$ \\
\hline Loss in SMF & $0.2 \mathrm{~dB} / \mathrm{km}$ \\
\hline Loss in DCF & $0.5 \mathrm{~dB} / \mathrm{km}$ \\
\hline Dispersion in SMF & $+17 \mathrm{ps} / \mathrm{nm}-\mathrm{km}$ \\
\hline Dispersion in DCF & $-85 \mathrm{ps} / \mathrm{nm}-\mathrm{km}$ \\
\hline Number of Loops & 20 \\
\hline Bit Rate & $10 \mathrm{~Gb} / \mathrm{s}$ \\
\hline Channel & 16 \\
\hline Channel Spacing & $0.8 \mathrm{~nm}$ \\
\hline
\end{tabular}

\section{SIMULATION RESULTS}

In this section, it is simulated the BER and $Q$ factor of the WDM system for long distance communication. These are the most important factors to evaluate the performance of the WDM optical network. Results obtained for both NRZ and RZ to observe the comparison performance of the predicted WDM model.

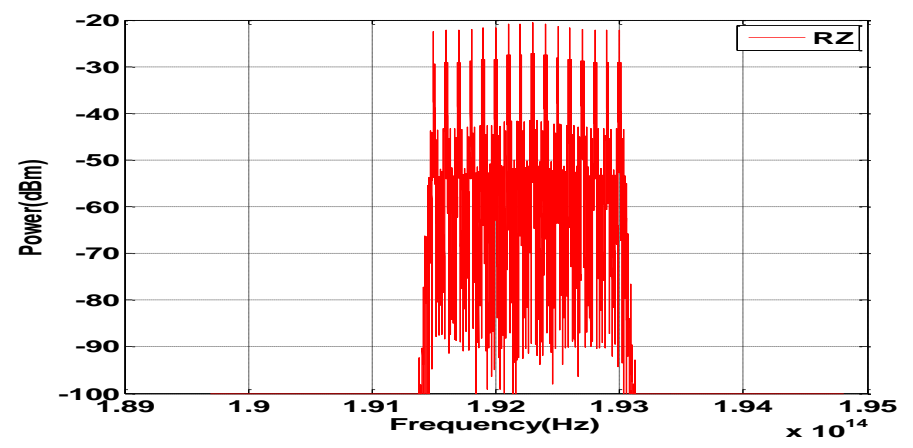

Fig.4: Optical Input Spectrum for 16 Channels in RZ modulation format

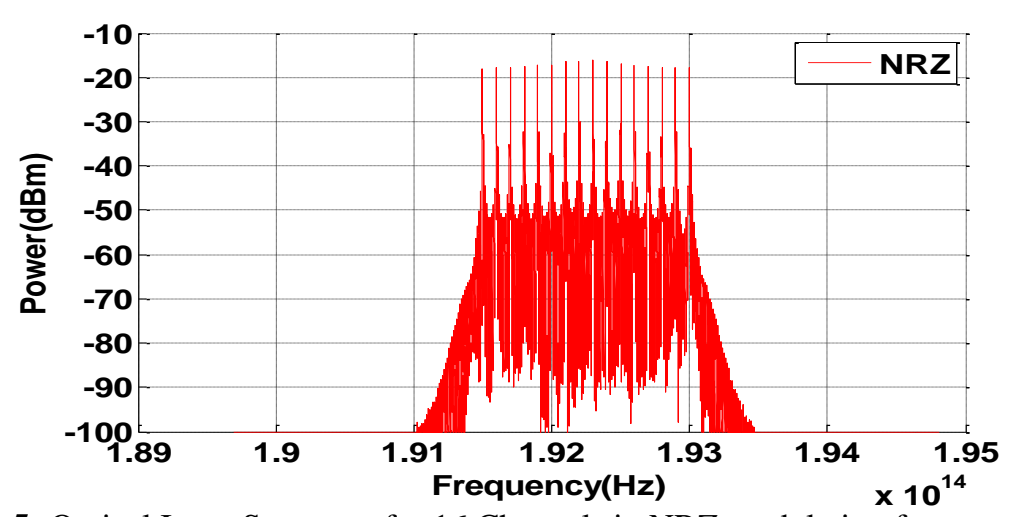

Fig.5: Optical Input Spectrum for 16 Channels in NRZ modulation format 
Fig.4 and Fig.5 present the WDM optical input spectrum considering input power (dBm) versus frequency $(\mathrm{Hz})$. It is generated for 16 channels for different frequency levels. The optical spectrum uses the above designed model. It is obtained from Optical spectrum analyzer. The wavelength that ranges from $1554.4 \mathrm{~nm}$ to $1566.5 \mathrm{~nm}$ is used in the WDM system. In Fig.5, the channels are denser for power level $-53 \mathrm{dBm}$ to downwards whereas this scenario is lighter in case of Fig.4. The power levels $-42 \mathrm{dBm}$ and $-52 \mathrm{dBm}$ are the points going to density of the channels in which the channels suffer channel spacing issue for RZ and NRZ modulation formats respectively

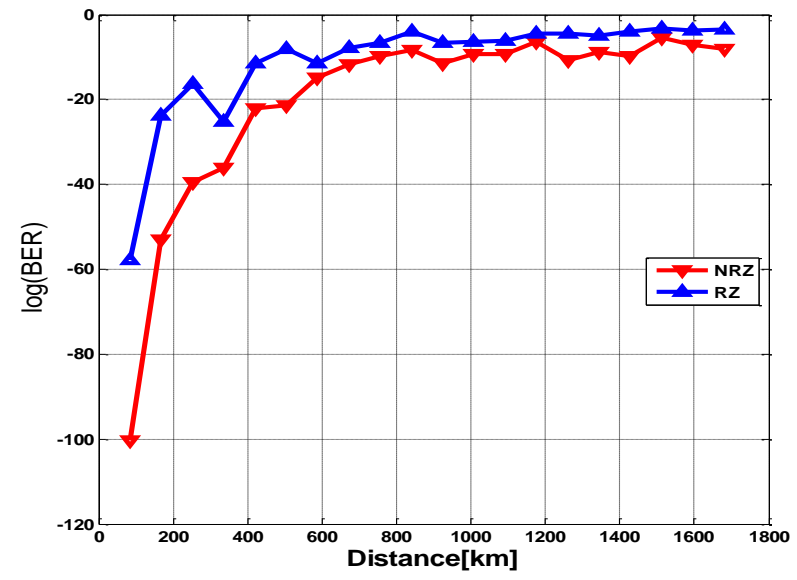

Fig. 6: BER versus distance for NRZ and RZ modulation formats

The bit error rate (BER) is simply the probability that an error will occur in a given bit period. Q-factor characterizes the quality of a digital signal from an analog point of view, therefore it is judged as a signal to noise ratio [20], [15]. Fig. 6 represents the comparison graph for NRZ and RZ modulation schemes for long distance communication. The lowest acceptable minimum log bit error found for NRZ is at $1500 \mathrm{~km}$ and the value is -5.42 . But for RZ, the lowest acceptable rate is achieved at $840 \mathrm{~km}$ and the value is -4.044 . Again the BER at $1600 \mathrm{~km}$ for NRZ encoding is -8.0571 and for RZ is -3.38 and this is not an acceptable BER for a signal transmission over this long distance.

It is observed that desired distance cannot be achieved if RZ modulation scheme is used. The reason behind this type of result is that RZ is more affected by dispersion than NRZ. RZ has shorter pulse width so it is affected by dispersion. Though NRZ is also affected by dispersion but NRZ is more affected by nonlinearity than dispersion. NRZ gives a better performance than RZ.

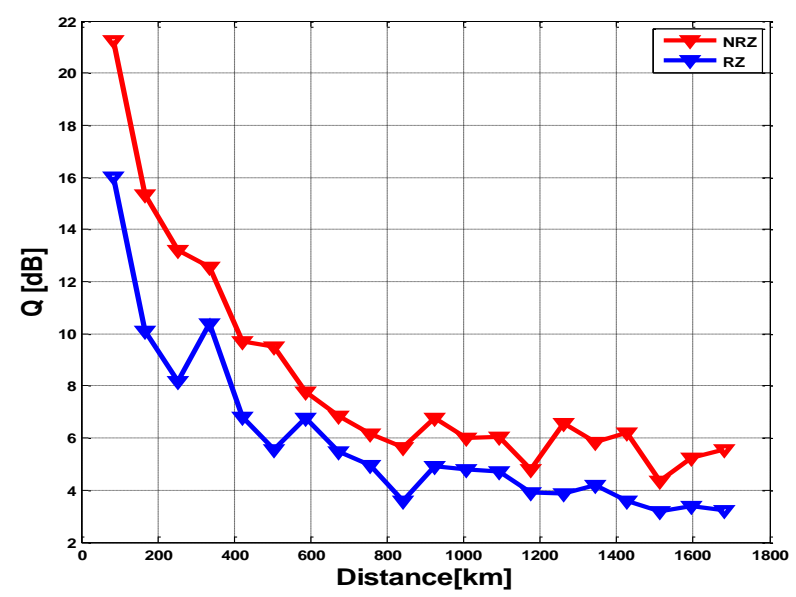

Fig. 7: Q factor versus distance for NRZ and RZ.

In Fig. 7, the variation of maximum $Q$ factor with distance is shown. Fig.7 shows that the $\mathrm{Q}$ factor becomes lower with the increment of the optical link distance. The $\mathrm{Q}$ factor has an inverse relationship with BER. It is for the same reason as previously discussed for BER. The value of maximum $Q$ factor at distance of $1600 \mathrm{~km}$ for $\mathrm{NRZ}$ is $5.5667 \mathrm{~dB}$ and for RZ is $3.2292 \mathrm{~dB}$ which is very low than NRZ format.The lowest Q-factor for NRZ is calculated at distance of $1500 \mathrm{~km}$ and the value is $4.362 \mathrm{~dB}$ and the lowest $\mathrm{Q}$ factor for $\mathrm{RZ}$ is $3.17 \mathrm{~dB}$ at $1500 \mathrm{~km}$. 


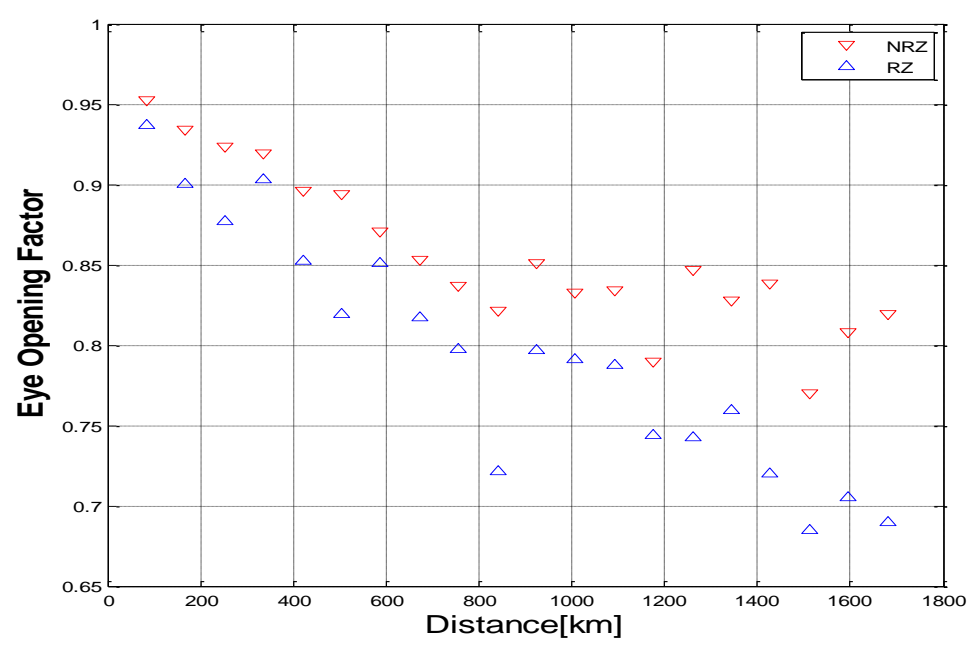

Fig. 8: Eye opening factor versus distance for NRZ and RZ.

Eye opening factor is the ratio of the height of the eye to the amplitude. Large eye opening factor represents the better performance of the system [21]. Fig. 8 presents the eye opening factor which varies with distance. In long distance fiber optic communication, it is really necessary to calculate the eye opening factor because it provides the condition of the signal or bits that has been transmitted. In Fig.8, it is found that NRZ gives better performance than RZ. The graph is not completely linear because the signal is affected by different types of noises and other types of attenuation for different optical link distance. At $1600 \mathrm{~km}$, the value of maximum eye opening factor for NRZ is 0.8204 and for RZ is 0.6903 . The lowest eye opening factor for NRZ is 0.7711 at the distance of $1500 \mathrm{~km}$ and for RZ the value is 0.6853 at $1500 \mathrm{~km}$.

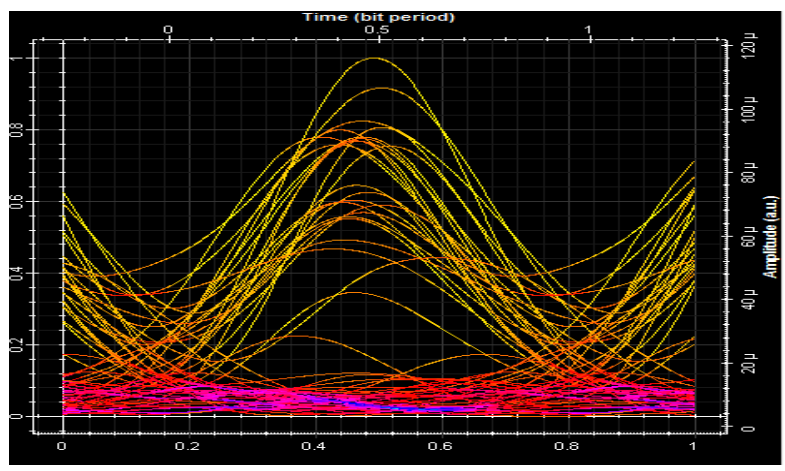

Fig. 9: Eye diagram of RZ format after $1680 \mathrm{~km}$ optical link distance considering channel number of 1

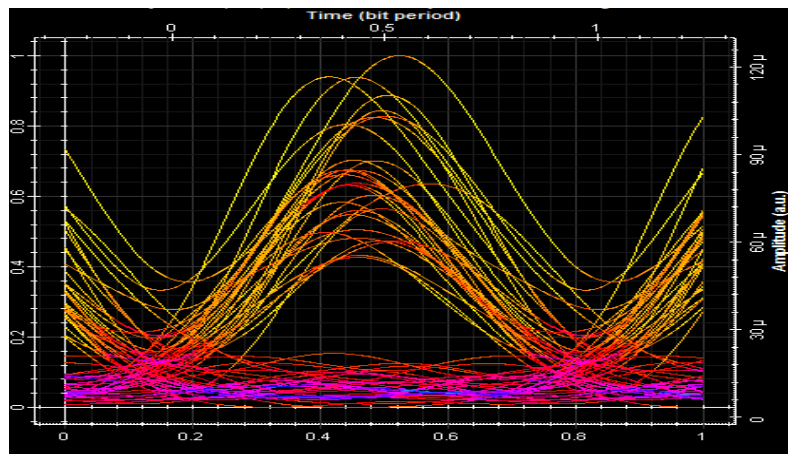

Fig. 10: Eye diagram of RZ format after $1680 \mathrm{~km}$ optical link distance considering channel number of 8 


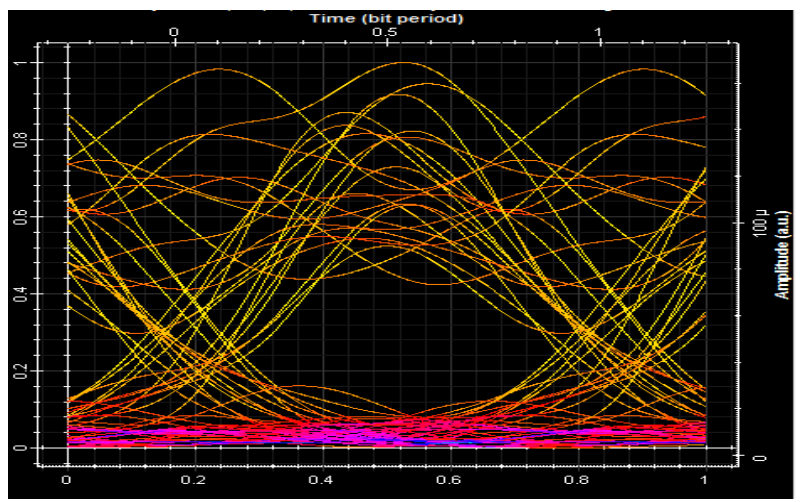

Fig. 11: Eye diagram of NRZ format after $1680 \mathrm{~km}$ optical link distance considering channel number of 1

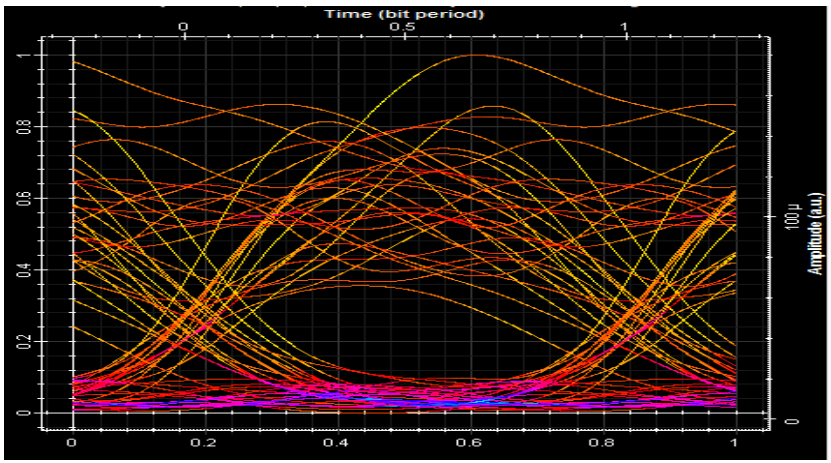

Fig. 12: Eye diagram of NRZ format after $1680 \mathrm{~km}$ optical link distance considering channel number of 8

Fig.9 and Fig.10 are the eye diagrams over 1680km WDM transmission for channel 1 and channel 8 using RZ modulation format respectively. In this format, it is found that the $\mathrm{Q}$ values are $3.22 \mathrm{~dB}$ and $3.18 \mathrm{~dB}$ for channel 1 and channel 8 respectively. Similarly, Fig.11 and Fig.12 are the eye diagrams over 1680km WDM transmission for channel 1 and channel 8 using NRZ modulation format respectively. In this modulation format it is examined that the $\mathrm{Q}$ values are $3.9 \mathrm{~dB}$ and $3.42 \mathrm{~dB}$ for channel numbers 1 and 8 respectively. The penalty of $\mathrm{Q}$ values for channel 1 considering RZ and NRZ is $0.68 \mathrm{~dB}$. The penalty of $\mathrm{Q}$ values for channel 8 is $0.24 \mathrm{~dB}$ considering RZ and NRZ format.

\section{CONCLUSION}

In this paper, the simulation investigation of 16x10Gbps WDM transmission for two different modulation techniques considering SMF and DCF commercial optical fiber types has been examined. In conclusion, it is also observed that the NRZ modulation has a better result than the RZ encoding for nonlinearity independent WDM transmission system. The BER, the Q factor and the eye opening factor show that the results are always better for NRZ encoding technique. It is found that NRZ modulation format carries out the better results than the RZ modulation format to obtain the higher quality values $(\mathrm{dB})$ and lower bit error rate (BER). It is also found that the quality factor is going downward with respect to optical link distance which is better than the RZ modulation format. In summary, NRZ is a good option for WDM communication system to operate large number of channels. NRZ encoding technique has the potentiality to overcome the limitations of long haul optical communication systems.

\section{REFERENCES}

[1] G. Bosco,A. Carena, V. Curri, R. Gaudino, and P. Poggiolini, "On the Use of NRZ, RZ, and CSRZ Modulation at $40 \mathrm{~Gb} / \mathrm{s}$ With Narrow DWDM Channel Spacing”, JLT, VOL. 20, NO. 9, SEPTEMBER 2002

[2] Monirul Islam, N. Ahmed, Sharafat Ali, S. A. Aljunid, R. B. Ahmad, Md. Shawkat Ali, "Hybrid (OCDMA/WDM) System with DPSK Modulation Using Different Detection Technique at Bit Rate 2.5Gbps for Optical Access Network", IJMOT, VOL.11, NO.4, JULY 2016

[3] Rajdi AGALLIU, "Comparison of Modulation Formats in Fiber-Optic Transmission Systems", POSTER 2015, PRAGUE MAY 14 
[4] Rupinder Kaur, Sanjeev Dewra, "Evaluation of Wavelength Division MultiplexingSystem using Different Modulation Formats", IJARCCE, Vol. 4, Issue 6, June2015

[5] Jinyu Mo, Yang Jing Wen, Yi Dong, Yixin Wang, Chao Lu, "Generation, detection and characterization of optical minimum shift keying data format”, Optics Communications 270, 396-401(2007).

[6] Wenke Yu, Li Huo, Dan Lu, Caiyun Lou, "A novel optical picosecond-duration NRZ-to-RZ format converter with simultaneous wavelength multicasting using a single-stage Mach-Zehnder modulator", Optics Communications 285,4302-4306(2012).

[7] Zhan-qiang Hui, Jia-min Gong, Meng Liang, Mei-zhi Zhang, Hui-min Wu, "Demonstration of all-optical RZ-to-NRZ format conversion based on self phase modulation in a dispersion flattened highly nonlinear photonic crystal fiber", Optics \& Laser Technology 54, 7-14 (2013).

[8] Hardeep Singh, Nivedita Mishra, "Performance Analysis of NRZ, RZ, Raised Cosine and GaussianModulation Formats in 32x10 Gbps WDM System with DifferentCompensation Techniques", Proc. SPIE 9579, Novel Optical Systems Design and Optimization XVIII, 95790U (September 3, 2015)

[9] Abu Sahmah Supa'at and Farah Diana Mahad, "EDFA Gain Optimization for WDM System," ELEKTRIKA, vol. 11, no. 1, pp. 34-37, 2009.

[10] S.Y. Park, H.K. Kim, C.S. Park, and S.-Y. Shin, "Doped fibre length and pump power of gain-flattened EDFAs," Electronics Letters, vol.32, no. 23, pp. 2161-2162, November 1996.

[11] R. W. Tkach, A. R. Chraplyvy, Fabrizio Forghieri, A. H. Gnauck, and R. M. Derosier, "Four-photon mixing and high-speed WDM systems," J. Lightwave Technol., vol. 13, pp. 841-849, May 1995.

[12] M.I. Hayee, A.E. Willner, "NRZ versus RZ in 10-40Gb/s Dispresion-Managed WDM transmission system," IEEE Photonics Technology Letters, Vol. 11, No. 8, August 1999.

[13] Douglas Walsh, David Moodie, Iain Mauchline, Steve Conner, Walter Johnstone, Brian Culshaw , "Practical Bit Error Rate Measurements on Fibre Optic Communications Links in Student Teaching Laboratories”. OptoSci Ltd, 141 St. James Rd., Glasgow, G4 OLT, Scotland,UK.

[14] NEBENDAHL, B., SCHMOGROW, R., DENNIS, T., JOSTEN, A., HILLERKUSS, D., KOENIG, S., MEYER, J., DRESCHMANN, M., WINTER, M., HUEBNER, M., FREUDE, W., KOOS, C., LEUTHOLD, J. Quality Metrics in optical modulation analysis: EVM and its relation to Q-factor, OSNR, and BER, Communications and Photonics Conference (ACP), 2012, p. 1-3.

[15] S. M. Jahangir Alam, M. Rabiul Alam, Guoqing Hu, and Md. Zakirul Mehrab, "Bit Error Rate Optimization in Fiber Optic Communications," International Journal of Machine Learning and Computing, Vol.1,No. 5,December2011.

[16] Supinder Kaur, Simarpreet Kaur, " Comparative Analysis between NRZ and RZ coding of WDM Systems in Optical Wireless Communication”, IJMECE, Vol. N0.4, Issue No.3, May,2016

[17] https://optiwave.com/resources/applications-resources/optical-system-comparison-of-rz-and-nrzmodulation-formats-for-40-gbs-systems/

[18] Karim F. George, Taher M. Bazan, Samy Ghoniemy and EI-Sayed A. El-Badawy, "Twelve-40 Gb/s WDM Communication Network with Different Modulation Formats", $6^{\text {th }}$ International Symposium on HONET, 2009

[19] Jasleen Kaur,Jyotsana and Jyoteesh Malhotra, “ Performance Comparison of 8x10Gbps WDM System Using RZ and NRZ Format”, IJSIP,Vol.8, No.11 (2015)

[20] Lei Ding, Wen De Zhong, Yixin Wang, and Yaolong Sam, “Optical Signal Quality Monitoring Method Based on Sub-harmonically Syncronus sampling” (ICOCN2002)” November 2002.

[21] http://www.yellowfourier.com/eyedia.html 